\title{
CLAVES PARA LA IDENTIFICACION DE MADERAS DE ARBOLES NATIVOS Y CULTIVADOS EN CHILE *
}

\author{
C. D. Oxf., 811.814
}

J. E. Díaz - Vaz O. **

\section{RESUMEN}

Las características macro y microscópicas más típicas de 43 maderas nativas y exóticas cultivadas en Chile, se han reunido sistemáticamente para confeccionar, dos claves dicótomas de identificación.

\section{SUMMARY}

The most representative macroscopic and microscopic characteristics from 43 chilean and exotic woods were species were employed to realise two dichotomous keys for identification.

\section{$1^{\circ}$ INTRODUCCION}

La madera presenta una gran variabilidad en sus propiedades y características la que le permite satisfacer distintos requisitos, teniendo por ello múltiples posibilidades de empleo. Si se considera, por ejemplo, el rango en que fluctúa su densidad, de 0,3 a $1,3 \mathrm{~g} / \mathrm{cm}^{3}$, se entiende que deben presentarse innumerables puntos de concidencia entre requisitos exigidos por distintos productos y las características de esta materia prima. La variabilidad en las características de la madera es consecuencia de las diferencias anatómicas específicas. Diferencias menores se presentan también dentro de cada especie e incluso en un mismo árbol. Como cada producto requiere madera con características específicas es fácil inferir la importancia de emplear en cada caso la especie más adecuada. De aquí la importancia y necesidad del reconocimiento de las maderas.

El reconocimiento anatómico de maderas pueden ser microscópicos y macroscópico, Aún cuando el primero es mucho más seguro que el segundo, requiere de equipos y técnicas especiales no siempre disponibles por el usuario. Deben entenderse como características macroscópicas de la madera, aquellas visibles a simple vista o con ayuda de un aumento pequeño,

* Proyecto F-2 financiado por la Vicerrectoría de Investigación de la Universidad Austral de Chile, 1975.

** Dr. Ingeniero Forestal, Profesor Director del Departamento de Tecnología e Industrias de la Madera, Universidad Austral de Chile, Casilla 567, Valdivia-Chile. cercano al $10 \mathrm{X}$. En la mayoría de los casos, estos caracteres macroscópicos tomados como un conjunto, son típicos para cada especie, permitiendo de este modo la identificación.

El presente trabajo considera ambos tipos de reconocimientos, entregando en forma separada claves dicótomas de identificación basadas en caracteres macroscópicos una y microscópicos la otra. Luego de las claves, se agregan explicaciones de los términos técnicos empleados y que son indispensables para el uso de ellas.

La clave "macroscópica" emplea fundamentalmente características primarias que son las menos variables dentro de cada especie, como por ejemplo, la presencia o ausencia de poros. Sin embargo han debido incluirse algunas características secundarias como el color y otras, cuando las primarias no son suficientes para tipificar todas las especies consideradas. En algunas especies pueden presentarse dudas al observar una característica; para disminuir esta posibilidad de error, la especie es incluida cada vez que es necesario, en las dos posibilidades planteadas por la clave. Por lo tanto, algunas especies aparecen citadas más de una vez.

\section{$2^{\circ}$ METODOLOGIA}

Las muestras empleadas en el presente trabajo corresponden al material que posee el Departamento de Tecnología e Industrias de la Madera de la Universidad Austral en Valdivia (Chile) y a recolecciones hechas por el autor. Gran parte de las preparaciones microtómicas pertenecen a la colección del mismo Departamento. Las observaciones a simple vista, bajo aumentos de $10 \mathrm{X}$ y las microscópicas se realizaron según los procedimientos que indica la literatura, cotejándose los datos obtenidos con los existentes en la bibliografía. (BROWN, PANSHIN and FORSAITH, 1949; INGLE and DADSWELL, 1953-1956; GREGUSS, 1955-1972; GOTTWALD， 1958; KRIBS， 1968; TAINTER, 1968; PATEL, 1973; BOSSHARD, 1974; DIAZ-VAZ, 1975).

Las probetas destinadas al análisis macroscópico fueron tratadas previamente con temperatura en condición de saturación, con agua- 
glicerina 50/50. Luego de cortar su superficie con el micrótomo, la mayoría recibió una impregnación con polietilenglicol, PG 600 al 30\% para evitar rajaduras $y$ otras deformaciones. Para las preparaciones con cortes microscópicos se utilizaron cubos de $1 \mathrm{~cm}$ de arista, los que fueron previamente saturados en aguaglicerina $50 / 50$ y luego sometido a calentamiento. Los cortes microtómicos de $20 \mu \mathrm{m}$ de espesor, se tiñeron con safranina y se montaron en Bálsamo de Canadá.

En la superficie de las probetas para observación macroscópica y en las preparaciones para observación microscópica, se midieron numerosos elementos celulares, determinándose tanto dimensiones transversales como longitudinales, apoyándose estas últimas enmaceraciones de cada especie.

\section{$3^{\circ}$ CLAVE DE IDENTIFICACION SEGUN CARACTERISTICAS MACROSCOPICAS *}

1 - Con poros

$1-\operatorname{Sin}$ poros

2- Poros ordenados en bandas

2 - Poros no ordenados en bandas

3- Poros reunidos en una sola banda tangencial inicial en cada anillo de crecimiento.

3 - Poros ordenados en más de una banda tangencial por anillo o en bandas radiales zigzagueantes.

4- Radios leñosos muy anchos, poros notoriamente más pequeños que el ancho de los radios leñosos. pequeños que el ancho de los radios leñosos. Quercus robur

4- Radios leñosos siempre de menor tamaño que el diámetro de los poros

Castanea sativa

5- Radios leñosos 5 ó más veces más anchos que los poros.

5 - Radios leñosos menos de 5 veces mas anchos que los poros.

6 - Radios leñosos de más de $3 \mathrm{~mm}$ de alto en las secciones tangenciales con engrosamiento muy notorio al termino de cada anillo de crecimiento. Gevuina avellana

6 - Radios leñosos menores de $3 \mathrm{~mm}$ en las secciones tangenciales y que no presentan generalmente un engrosamiento bien notorio al termino de cada anillo.

7- Madera de color café claro a veces azul grisáceo con bandas longitudinales oscuras.

Lomatia hirsuta

7- Madera de color café claro o rosado pálido que no presenta manchas oscuras.

Embothrium coccineum

2

3

4

5

(ENCINA)

(CASTAÑO)

6

8

(AVELLANO)

( RADAL)

(NOTRO)

8- Madera de color café oscuro con gran cantidad de tejido de color claro que le da a simple vista un aspecto jaspeado a las secciones longitudinales. El tejido de color claro tiende a formar bandas que en parte rodea a los poros.

Acacia caven

8- Madera que no presenta color café oscuro, ni tejido de color más claro que le de un aspecto jaspeado en las secciones longitudinales.

9- Banda marginal de color más claro siempre presente, bien delimitada.

Cryptocarya alba

9- Banda marginal de color más claro que el resto de la madera no siempre presente.
(ESPINO)

9

(PEUMO)

10

* Para observar las superficies, especialmente las secciones transversales, la muestra de madera debe ser humedecida y luego alisada con una herramienta cortante muy afilada. Para este fin es apropiada una hoja de afeitar. 
10 - Poros como canalículos visibles a simple vista y notorios al tacto en las secciones longitudinales. Radios leñosos muy delgados, de ancho menor a 10 veces el diámetro de los poros.

Eucalyptus globulus

10 - Poros que no se aprecian a simple vista como canalículos ni son detectables al tacto en las secciones longitudinales de ancho hasta diez veces menores que el diámetro de los poros.

11- Radios leñosos de ancho semejante o algo mayor que el diámetro de los poros.

Dasyphyllum diacanthoides

11 - Radios leñosos notoriamente benores al diámetro de los poros.

Lithraea caustica

12- Madera que a simple vista presenta tejido de color más claro en manchas $\mathrm{y} / \mathrm{o}$ bandas, diseminado en todo el anillo.

12- Madera que a simple vista no presenta tejido de color claro diseminado en todo el anillo.

13 - Madera de color café muy oscuro, muy pesada y dura.

13- Madera que no tiene color café muy oscuro.

14 Tejido de color más claro que el resto de la madera muy abundante, rodeando los poros y formando bandas confluentes. Ancho de los radios leñosos hasta tres veces menores que el diámetro de los poros. Acacia caven

14 Tejido de color claro no muy abundante que no forma bandas confluentes notorias que unen a los poros. Radios leñosos de ancho menor a tres veces el diámetro de los poros.

Prosopis tamarugo

15- Radios leñosos en sección tangencial como manchas elípticas pequeñas más oscuras que el resto del tejido, fáciles de ver bajo la lupa. Madera de color café claro, con tintes verdosos.

Iithrea caustica

15- Radios leñosos que no se aprecian claramente como manchas mucho más oscuras en sección tangencial. Madera de color café pálido, amarillento. Dasyphyllum diacanthoides

16- Madera de color verde oscuro con tinte azul con bandas de tinte café rojizo*.

Porlieria chilensis

16- Madera que no tiene un color verde oscuro con bandas de tinte café rojizo amarillento.

17- Ancho de los radios leñosos levemente mayores que el diámetro de los poros.

17- Ancho de los radios leñosos semejante o menor tamaño que el diámetro de los poros.

18 - Madera de color café rojizo oscuro con estrías negras delgadas alargadas longitudinalmente. Weinmania trichosperma

18 - Madera que no presenta una coloración café rojiza oscura ni bandas negras, delgadas, alargadas longitudinalmente.
(EUCALIPTO)

(ESPINO)

(TAMARUGO)

(GUAYACAN)

17

(TINEO)

\footnotetext{
* Madera muy dura y pesada. Muy difícil de cortar.

La albura de esta madera es de color amarillo

verdoso lo que contrasta muy fuerte con el dura-

men verde oscuro. Los radios leñosos son total-

mente estratificados en pisos pero muy difícil de

observarlos, ya que son muy pequeños.
} 
19-Madera de color grisáceo amarillenta*.

\section{Laurelia philippiana}

19- Madera que no presenta color grisáceo amarillento.

20- Radios leñosos muy numerosos y de ancho notoriamente mayor que el diámetro de los poros. Madera de color café oscuro.

Peumus boldo

20 - Radios leñosos más escasos de ancho igual o menor que el diámetro de los poros. Madera que no presenta color café oscuro.

21- Poros escasos bien visibles y aislados que presentan una reducción fuerte del diámetro inicial hacia el término del anillo. Madera de color café muy pálido, grisáceo, con tinte amarillento.

Quillaja saponaria

21 - Poros difíciles de ver, que no muestran una reducción notoria del diámetro inicial hacia el término del anillo. Madera sin color café pálido, grisáceo, y de tinte amarillento.

22- Madera que en sección tangencial presenta radios leñosos como manchas pequeñas, oscuras, bien notorias, que le dan un aspecto jaspeado.

Aextoxicon punctatum

22- Madera que no presenta radios leñosos, como manchas pequeñas, oscuras, que le dan un aspecto jaspeado a la sección tangencial.

23- Poros y radios leñosos muy pequeños, apenas visibles a la lupa. Madera de color café pálido con tinte grisáceo.

Myrceugenella apiculata

23- Poros y radios leñosos más grandes visibles a la lupa Madera de color café rosado pálido. Caldcluvia paniculata

24 Madera con canalículos muy notorios a simple vista y al tacto con aspecto de rasguños en sección longitudinal. Poros varias veces menores que los radios leñosos, escasos $\mathrm{y}$ distribuidos en todo el anillo.

24 Madera con canalículos poco notorios a simple vista con aspectos de rasguños en sección longitudinal. Poros de tamaño semejante a los radios leñosos, que pueden ser numerosos y no distribuidos en todo el anillo.

25- Madera de color café rojizo muy oscuro, con bandas longitudinales negruzcas.

Prosopis tamarugo

25- Madera que no presenta color muy oscuro ni bandas longitudinales negruzcas.

26- Anillos anchos, bien delimitados. Poros muy visibles, aislados y escasos.

Acacia melanoxylon

26- Anillos angostos no siempre bien delimitados. Poros visibles más abundantes.

Persea lingue

27- Madera con banda marginal delgada, de color más claro bien notoria y delimitada siempre presente**.

27- Madera que no presenta regularmente una banda marginal de color claro en cada anillo.

\footnotetext{
Anillos de crecimiento muy difíciles de delimitar

bajo la lupa. Madera de duramen con manchas café muy oscuras de olor fuerte y desagradable.
}

(TEPA)

20

(BOLDO)

(QUILLAY)

(OLIVILLO)

(ARRAYAN)

(TIACA)

(TAMARUGO)

26

(AROMO AUSTRALIANO)

(LINGUE)

28

31
** En los cortes longitudinales tangenciales los radios leñosos se ven como manchitas estratificadas en pisos. 
28 - Madera de olor ácido a vinagre que por lo general presenta estrías filamentosas zigzagueantes en dirección longitudinal en sección tangencial. Madera con visos muy suaves de tonos pardos.

Nothofagus dombeyi

28- Madera que no presenta estrías longitudinales, sin olor ácido a vinagre.

29 - Madera de color rojo oscuro *.

29- Madera que no presenta color rojo oscuro.

Nothofagus obliqua

30 - Banda marginal de color claro no siempre presente, Madera de color café rosado pálido, de veteado suave a liso.

Nothofagus alpina

30 - Banda marginal de color más claro siempre presente. Madera de color café pardo pálido con manchitas oscuras que le dan un aspecto jaspeado en sección tangencial.

31- Madera de color rojo oscuro

31- Madera que no presenta color rojo oscuro.

Cyptocarya alba Nothofagus obliqua

32- Madera tardía bien definida, sin poros y de tono más oscuro que la madera temprana. Poros solitarios muy numerosos más o menos aislados. Madera de color café pardo oscura muy pesada.

Amomyrtus luma

32- Madera tardía poco definida, con poros y de tono semejante a la madera temprana. Poros solitarios escasos. Madera de peso moderado de color claro.

33- Madera de color verdoso con tintes café amarillentos y de veteado liso.

Laurelia sempervirens

33- Madera que no es de color verdoso ni con tintes café amarillentos, de veteado suave.

34 Radios leñosos muy delgados con anchos bastantes menores que el diámetro de los poros.

34 Radios leñosos de ancho semejante o algo menores que el diámetro de los poros.

35- Madera con olor característico muy semejante a vinagre Sección tangencial con marcas filiformes, onduladas en dirección longitudinal.

Nothofagus dombeyi

35- Madera sin olor ácido a vinagre ni marcas en sección tangencial.

36- Los radios leñosos visibles a la lupa como manchitas notoriamente más oscuras que el resto de la madera en sección tangencial. Madera dura de color café y tintes verdosos.

Lithraea caustica

36- Radios leñosos no se ven como manchitas notoriamente más oscuras que el resto de la madera, sin tintes verdosos.

37- Madera de color amarillo grisácea muy liviana y blanda. Populus nigra

37- Madera de color café rosado, pálido moderadamente pesada y dura.

Nothofagus pumilio
(COIGUE)

29

(ROBLE)

30

(RAULI)

(PEUMO)

(ROBLE)

32

(LUMA)

(LAUREL)

34

35

38

(COIGUE)

36

(LENGA)

\footnotetext{
* Veteado liso, anillos no siempre fáciles de delimitar $\mathrm{y}$ generalmente sin banda marginal de tejido de color claro.
} 
38- Madera de color café rojizo pálido*.

38 - Madera que no presenta color rojizo pálido.

39- Madera de color café muy pálido, con tinte amarillo verdoso.

39- Madera sin tinte verdoso amarillento.

Lithraea caustica

40 - Poros en gran proporción solitarios y aislados. Madera de color café amarillento muy pálido, algo grisáceo.

Quillaja saponaria

40 - Poros solitarios y aislados en escasa proporción. Madera sin color amarillento grisáceo.

41- Poros visibles a la lupa que disminuyen en diámetros desde la madera temprana a la tardía. Eucryphia Cordifolia

41- Poros muy pequeños difíciles de distinguir a la lupa y que no varían en diámetro desde la madera temprana a la tardía.

Myrceugenella apiculata

42- Radios leñosos muy delgados, difíciles de distinguir.

42- Radios leñosos notoriamente multiseriados a simple vista.

43 - Canales resiníferos presentes.

Drymis winteri

43- Canales resiníferos ausentes.

44 Transición brusca de la madera temprana a la madera tardía en un mismo anillo. Madera liviana de color café pálido Pseudotsuga menziesii

44 Transición suave de madera temprana a la tardía en un mismo anillo. Madera liviana de color café amarillento pálido. Generalmente presenta mancha azul.

Pinus radiata

45- Madera de olor fuerte, semejante a la vainilla, persistente y algo picante.

Pilgerodendron uviferum

45- Madera que no presenta olor fuerte, persistente, semejante a vainilla.

46- Madera de color café rojizo muy oscura**.

Fitzroya cupressoides

46- Madera que no presenta color café rojizo muy oscura.

47- Madera con aspecto jaspeado muy notable en sección tangencial originado por los radios leñosos de color más oscuro.

Araucaria araucana

47- Madera que no presenta un aspecto jaspeado muy notable en sección tangencial.

48 - Madera de color café pálido, con una banda terminal muy delgada de madera tardía más oscura que delimita muy bien los anillos de crecimiento. Austrocedrus Chilensis

48- Madera de color café, amarillento que no presenta una banda muy delgada terminal de madera tardía que delimite claramente los anillos de crecimiento.

Saxegothaea conspicua y Podocarpus sp
(RAULI)

39

(LITRE)

40

(QUILLAY)

41

(ULMO)

(ARRAYAN)

(CANELO)

44

45

(PINO OREGON)

(PINO INSIGNE)

(CIPRES DE LAS GUAY-

TECAS)

46

(ALERCE)

47

(ARAUCARIA)

48

(CIPRES DE LA CORDILLERA)

(MAÑIOS Y LLEUQUE)
* Radios leñosos con tendencia a estratificarse en pisos, en la sección tangencial. ** Anillos de crecimiento generalmente delgados, on-
dulados y bien delimitados por la madera tardía oscura. 


\section{$4^{\circ}$ CLAVE SEGUN CARACTERISTICAS MICROSCOPICAS}

1 - Madera con poros.

1 - Madera sin poros.

2- Radios leñosos múltiples de varias células. Drymis winteri

2- Radios leñosos casi exclusivamente uniseriados.

(CANELO)

3- Canales resiníferos presentes.

3 - Canales resiníferos ausentes.

4

5

4- Engrosamientos espiralados presentes. Pseudotsuga menziesii

4 - Engrosamientos espiralados ausentes Pinus radiata

(PINO OREGON)

(PINO INSIGNE)

5- Campo de cruces con una punteadura que es del tipo ventaniforme.

Podocarpus andinus

5- Campo de cruces con más de una punteadura.

\section{(LLEUQUE)}

6

(ARAUCARIA)

7

8

10

7- Campo de cruces con más de tres punteaduras.

8- Radios leñosos de 15 a 20 células de altura, bastante frecuentes, generalmente de 1 a 10 células. Podocarpus nubigenus

8 - Radios leñosos de 15 a 18 células de altura, muy escasos, generalmente de 1 a 10 células.

9- Generalmente una punteadura por campo de cruces.

Podocarpus salignus (MAÑIO DE HOJAS

9- Generalmente una o dos punteaduras por campo de cruces.

\section{Saxegothea conspicua (MAÑIO HEMBRA)}

10 - Radios leñosos con 2 a 6 células de altura.

10 - Radios leñosos con 1 a 15 células de altura.

Fitzroya cupressoides

11- Parénquima longitudinal muy abundante.

\section{Pilgerodendron uviferum}

11- Parénquima longitudinal ausente o escaso.

Austrocedrus chilensis

12- Maderas con porosidad difusa.

12- Poros ordenados en bandas tangenciales $\mathrm{u}$ oblicuas.

13- Poros en una banda tangencial.

13 - Poros en varias bandas tangenciales.

Castanea sativa

14 Radios leñosos uni a triseriados.

Quercus robur
LARGAS )

(MAÑIO MACHO)

9

(ALERCE)

11

(CIPRES

DE LAS GUAYTECAS)

(CIPRES DE LA CORDILLERA)

20

13

14

15

(CASTAÑO)

(ENCINA) 
15- Poros en bandas oblicuas radiales o tangenciales, zigzagueantes.

15- Poros en bandas tangenciales no zigzagueantes. Madera de porosidad semicircular.

16- Engrasamiento espiralados presentes.

Gevuina avellana

16- Engrosamientos espiralados ausentes.

(AVELLANO)

17

17- Radios leñosos uniseriados, escasos. Embothrium coccineum

(NOTRO)

17- Radios leñosos uniseriados, abundantes. Lomatia hirsuta

( RADAL)

18 - Engrosamientos espiralados muy notorios.

Dasyphyllum diacanthoides

18 - Engrosamientos espiralados poco notorios o ausentes.

(TREVO)

19- Poros solitarios, de gran tamaño, hasta $350 \mu \mathrm{m} / \mathrm{de}$ diámetro. Eucalyptus globulus

19- Poros solitarios y múltiples de hasta 6 células, pequeños de diámetros menores a $100 \mu \mathrm{m}$. Lithraea caustica

20- Radios leñosos exclusivamente uni a biseriados.

20 - Radios leñosos multiseriados, de más de 2 células.

21- Poros exclusivamente solitarios. Parénquima apotraqueal en bandas delgadas distribuidas en todo el anillo.

Porlieria chilensis

21- Poros solitarios y múltiples. Parénquima apotraqueal en bandas delgadas ausentes.

22- Engrosamiento espiralados presentes. Nothofagus pumilio

22- Engrosamientos espiralados ausentes.

23 - Placas de perforaciones escalariformes ausentes.

23 - Placas de perforaciones escalariformes presentes.

24 Radios leñosos heterogéneos tipo I de Kribs con máximo de 30 células de altura.

24 Radios leñosos heterogéneos tipo III de Kribs con máximo de 16 células de altura.

25- Radios leñosos hasta 35 células de altura. Nothofagus alpina

25- Radios leñosos hasta 25 células de altura. Eucryphia cordifolia

(ULMO)

26- Radios leñosos uniseriados, los biseriados son escasos o están ausentes.

26- Radios leñosos biseriados en gran proporción.

Nothofagus obliqua

28 - Placas de perforaciones exclusivamente escalariformes.

28 - Placas de perforaciones escalariformes ausentes.

29- Radios leñosos con altura máxima de 20 células

\section{Laurelia sempervirens}

29- Altura máxima de radios leñosos mayor a 25 células.

30 - Radios leñosos con más de 4 células de ancho. 
31- Engrosamientos espiralados a veces presentes.

\section{Aextoxicon punctatum (OLIVILLO)}

31- Engrosamientos espiralados ausentes.

Caldcluvia paniculata

( TIACA)

32- Radios leñosos heterogéneos tipo I de Kribs.

\section{Weinmannia trichosperma (TINEO)}

32- Radios leñosos heterogéneos tipo II de Kribs.

33 - Radios leñosos numerosos, 20 por $\mathrm{mm}$.

\section{Myrceugenella apiculata (ARRAYAN)}

33 - Radios leñosos poco numerosos, 10 ó menos por $\mathrm{mm}$.

\section{Laurelia philippiana}

34 Radios leñosos, mayores a $1500 \mu \mathrm{m}$. de altura.

(TEPA)

34- Radios leñosos menores a $1500 \mu \mathrm{m}$ de altura.

35

36

35- poros casi exclusivamente solitarios.

Quillaja saponaria

35- Poros en su mayoría múltiples. Peumus boldo

(QUILLAY)

(BOLDO)

36- Parénquima paratraqueal vasicéntrico, confluente muy abundante.

36- Parénquima paratraqueal vasicéntrico, confluente, escaso o abundante.

37- Radios leñosos heterogéneos.

37- Radios leñosos homogéneos.

Amomyrtus luma (LUMA) 38

38- Radios leñosos uni a triseriados

Prosopis tamarugo

Acacia caven

38 - Radios leñosos de hasta 5 células de ancho.

39- Engrosamientos helicoidales presentes, algo difíciles de
apreciar. apreciar.

Lithraea caustica

39 - Engrosamiento helicoidales ausentes.

40 - Poros múltiples de más de 3 células, siempre presentes.

40 - Poros múltiples de hasta 3 células, escasos.

41- Placas de perforaciones escalariformes presentes.

41 - Placas de perforaciones escalariformes ausentes.

Nothofagus alpina

Nothofagus obliqua

43- Parénquima apotraqueal marginal, ancho siempre presente Cryptocarya alba

42- Parénquima apotraqueal marginal ausente.

43- Radios leñosos con alturas mayores de 35 células.

\section{Acacia melanoxylon}

43 - Radios leñosos con alturas no mayores de 30 células.

Persea lingue
(TAMARUGO)

(ESPINO)

40

42

(RAULI)

(ROBLE)

(PEUMO)

(AROMO AUSTRALIANO)

(LINGUE) 
$5^{\circ}$ GLOSARIO

Canalículos: Vasos que al ser cortados longitudinalmente, se aprecian como canales delgados en las secciones tangenciales y radiales.

Canal resinífero: espacio intercelular tubular dejado por células secretoras que forman un anillo alrededor de él.

Campos de cruces: rectángulo que se forma en el plano radial longitudinal, delimitado por las paredes de una célula alargada verticalmente y una célula alargada radialmente.

Engrosamiento helicoidal: protuberancia filiforme dispuesta espiralmente en la cara interna de la pared celular.

Estrías: ver canalículos.

Parénquima: elementos celulares almacenadores cortos, de paredes delgadas y generalmente con punteaduras simples.

Parénquima apotraqueal: parenquima ordenado independiente de la ubicacion de los poros, que se puede observar en las secciones transversales de la madera.

Parénquima marginal: parénquima que tiende a formar una banda tangencial al inicio o término de cada anillo, que se aprecia en la sección transversal de la madera.

Parénquima paratraqueal vasicentrico: parénquima que se presenta ordenado rodeando parcial o totalmente a un vaso, como una vaina, al observar las secciones transversales de la madera.

Parénquima confluente: parénquima que se extiende desde un poro a otro, uniéndolos.

Placa de perforación: área de contacto entre las paredes terminales de células unidas longitudinalmente que se encuentra perforada.

Placa de perforación escalariforme: placa de perforaciones alargadas y paralelas entre sí, denominándose barras a los restos de pared celular entre las perforaciones.

Poro: sección transversal de una traquea. Denominación que reciben los vasos en la sección transversal de la madera.

Poro solitario: en la sección transversal se aprecia cada uno de ellos separado del resto.

Poro múltiple: grupo de dos o más poros reunidos en fila como una cadena. Están apretados dejando sus paredes de contacto aplanadas, de forma que aparecen como subdivisiones de un poro de mayor tamaño.

Poros agrupados: grupo de tres o más poros reunidos en racimos y apretados, con sus paredes de contacto aplanadas.

Porosidad: conjunto de poros en un anillo de crecimiento.

Porosidad difusa: poros que no presentan ordenación alguna en el anillo.

Punteadura: interrupción de la pared secundaria de la célula, pero no de la pared primaria y lámina media.

Punteadura aereolada: la cavidad producida por la interrupción es desigual, estrechándose hacia el lumen de la célula.

Punteadura simple: la cavidad producida por la interrupción es pareja en toda la pared secundaria.

Punteadura ventaniforme: en el campo de cruces las punteaduras se aprecian como rombos grandes de cantos redondeados.

Punteadura escalariforme: punteadura alargada dispuesta en series paralelas entre sí.

Radios leñosos: tejido en forma de cinta originado por el cambium y que se extiende en dirección radial.

Radios leñosos uniseriados: con una sola célula de ancho al observarlo en la sección longitudinal tangencial.

Radios leñosos multiseriados: con más de una célula de ancho al observarlos en la sección longitudinal tangencial.

Radios leñosos heterogéneos: conjunto de radios leñosos compuesto de células alargadas horizontal y verticalmente.

Radios leñosos heterogéneos tipo I de Kribs: en este tipo los uniseriados están formados por células alargadas verticalmente. Los multiseriados presentan en los extremos una parte uniseriada con células marginales alargadas verticalmente y que es mayor que la parte central multiseriada.

Radios leñosos heterogéneos tipo II de Kribs: los radios uniseriados están formados por células alargadas verticalmente. Los radios multiseriados son de extremos uniseriados más cortos que la parte central multiseriada y con células muy altas, alargadas verticalmente.

Radios leñosos heterogéneos tipo III de Kribs: los radios uniseriados son de células alargadas verticalmente, y de células alargadas horizontalmente. Los radios multiseriados presentan una sola célula marginal y alargada verticalmente.

Tejido de color claro: ver Parénquima.

Traquea: ver Vaso.

Vaso: célula de paredes delgadas y lumenes amplios, que se unen verticalmente entre ellas formando tubos muy largos. 
R E F E R E N C I A S

BOSSHARD, H.H., 1974. Holzkunde Band I Mikroskopie und Makroskopie des Holzes. Birkhäuser Verlag. Easel, 224 p.

BROWN, H.P., PANSHIN, A.J., FORSAITH, C.C., 1049. Texbook of Wood Technology. Vol. I Mc. Graw-Hill Boox Company, Inc. New York, Toronto London, $652 \mathrm{p}$.

DIAZ-VAZ, J.E., 1975. Identificación de Maderas, Charlas y Conferencias Nr. 3, Facultad de Ingeniería Forestal, Universidad Austral de Chile, 34-43.

GOTTWALD, H., 1958. Handelhölzer, Ferdinand Holzmann Verlag-Hamburg, 251 p.

GREGUSS, P., 1955. Xylotomische Bestimmung der Heute Lebenden Gymnospermen, Akademiai Kiado Budapest, 668 p.

GREGUSS, P., 1972. Xylotomy of the Living Conifers Awademiai Kiado, Budapest, $668 \mathrm{p}$.

INGLE, H.D., DADWELL, H.E., 1953. The Anatomy of the timbers of the southwest Pacific Area III Myrtaceae, Australian Journal of Botany, 1(5): 353-401.

INGLE, H.D., DADSWELL, H.E., 1956. The anatomy of the timbers of the southwest Pacific Area IV Cuoniaceae, Davidsoniaceae and Eucryphiaceae, Australian Journal of Botany, 4(2): 125-151.

KRIBS, D.A., 1968. Commercial Foreign Woods on the American Market, Dover Publications, Inc., New York, 241 p.

NEIRA, M., MARTINEZ MATA, F., 1973. Terminología Forestal Española. Instituto Nacional de Investigaciones Agrarias, Ministerio de Agricultura, Madrid, $480 \mathrm{p}$.

PATEL, R.N., 1973. Wood Anatomy of the Dicotyledons Indigenous to New Zealand 3: Monimiaceae and Atherospermataceae. New Zealand Journal of Botany 11:587-598.

TAINTER, F.H. La identificación microscópicas de las Maderas comerciales chilenas. Montana Forest and Conversation Experiment Station, School of Forestry, University of Montana Missoula, $27 \mathrm{p}$.

WAGEMANN, W., 1949. Maderas chilenas contribución a su anatomía e identificación. Actas del II Congreso Sudamericano de Botánica, Tucumán, 263-375. 